\title{
Hierarchical Cluster Analysis on Potential Resource of Nitrogen Fixing Bacteria at Wheat Monocrop Production Districts of Bale,Ethiopia
}

\author{
Wendesen Melak \\ Ethiopian Institute Of Agricultural Research,Kulumsa Agricultural Research Center, \\ Assela, Ethiopia P. O.Box 489
}

\begin{abstract}
Wheat monocrop production system at south eastern highlands of Ethiopia is a threat to productivity and adoption of high value highland pulses such as Faba bean which are the most important pulse crop in Ethiopia. Thirty faba bean nodulating isolates trapped from soil collected from twenty eight locations of Bale zone were characterized for possible diversity.For these purposes numerical taxonomy by multivariate cluster analysis based on physiological characteristics was used and according to which entire test isolates were grouped .Dendrogram constructed from cluster analysis of 56 phenotypic traits, grouped them into three clusters and one un-clustered positions at 53\% relative similarity. Cluster III contained 58\% of the test isolates that were grouped together with the reference strains Rhizobium leguminosarum (FBEAL-110), suggesting that besides to cmmonly nodulated on the study area were also phenotypically diverse. Furthermore, the result indicates the existence of strains in the collection, which can tolerate environmental stresses, thus can be developed into inoculant for highland pulses' inoculation and production in Ethiopia and beyond.
\end{abstract}

Keywords: Cluster analysis, Monocropping, Rhizobium leguminosarum

DOI: $10.7176 / \mathrm{JBAH} / 9-19-02$

Publication date:October $31^{\text {st }} 2019$

\section{Introduction}

Ethiopia is the second- largest producer of faba beans after China (ECX, 2009;IFPRI, 2010; Tamene Temesgen et al., 2015) there is still untouched potential in the country. Moreover, Ethiopia's average bean yield is very low when compared to other developed countries. This poor productivity coupled with small per capita land holding of the majority of farmers is a challenge to increasing production and the marketable surplus of bean(ECX, 2009)but potential producer districts are trapped with monocropping production system that affected pulse production and adoption rate.

The symbiotically fixed N2 fixing organisms by the association between Rhizobium species and the legumes, represents a renewable source of $\mathrm{N}$ for agriculture (Zahran, 2001).A symbiotic relationship is usually a highly specific process between fine plant host and the bacteria. (Fisher and Newton, 2002) in that regard potential of indigenous nitrogen fixing strain will turn considerable sum of diversity into utilizable that in its way impact soil fertility ,health and crop diversification.

\section{Materials and Methods}

sampling and trapping of isolates

Soil samples from farmers field were collected from 28 locations of Bale zone and trapped bacteria isolates were isolated following standard procedures. Plates were incubated at $28 \pm 20 \mathrm{C}$ for 3-5 days then restreaked to obtain pure culture. Single colony isolates were picked from plates, numbered and stored in YEMA slants containing $0.3 \%(\mathrm{~W} / \mathrm{V}) \mathrm{CaCO} 3$ at $40 \mathrm{C}$ refrigerator for further characterization(Somasegaran and Hoben, 1994;Howieson and Dilworth, 2016)

\section{Stress and nutrient utilization test}

Different rates of environmental stresses and utilization of different sources of Carbon and Nitrogen were qualitatively taken to to draw correlation matrix and phenogram

\section{Numerical Data Analysis}

Phenotypic similarities among 30 isolates and one reference strain(FBEAL-110) were numerically analyzed based on their phenotypic characteristics, such as $\mathrm{pH}$ tolerance, salt tolerance, temperature tolerance, Intrinsic antibiotic resistance, Carbon source and Amino acid utilization, using a similarity coefficient and phenogram was constructed by the hierarchical cluster analysis (HCA) based on agglomerative clustering which is the most common type of hierarchical clustering used to group objects in clusters based on their similarity(Kassambara, 2017) and appropriates R packages(factoextra \& dendextend) were used(R Core Team, 2019) 


\section{Results and Discussions}

Production of pulse crops has been improved by potential rhizobia when nitrogen fixation is efficient (Simon et al., 2014). The result indicated diverse groups of rhizobia in sampled districts. Our strains fall into fast growing Rhizobia based on doubling time, yellow color indicating the acid producing ability of the isolates on BTBYEMA.This result is consistent to results of other researchers on fast growing Faba Bean nodulating isolates(Abere Mnalku et al., 2009;Zerihun Belay and Fassil Assefa, 2011;Getahun Negash 2015).

Moreover generation times of 2-4hours with large colony diameter of 1-5mm (data not shown)and production of copious exopolysaccharide has put the isolates into cross-nodulation group of Rhizobium leguminosarum var. viceae(Somasegaran and Hoben, 1994;Berkum; et al., 1995).In addition to that they preliminarily responded similar to the already authenticated and commercial elite strain FBEAL-110.

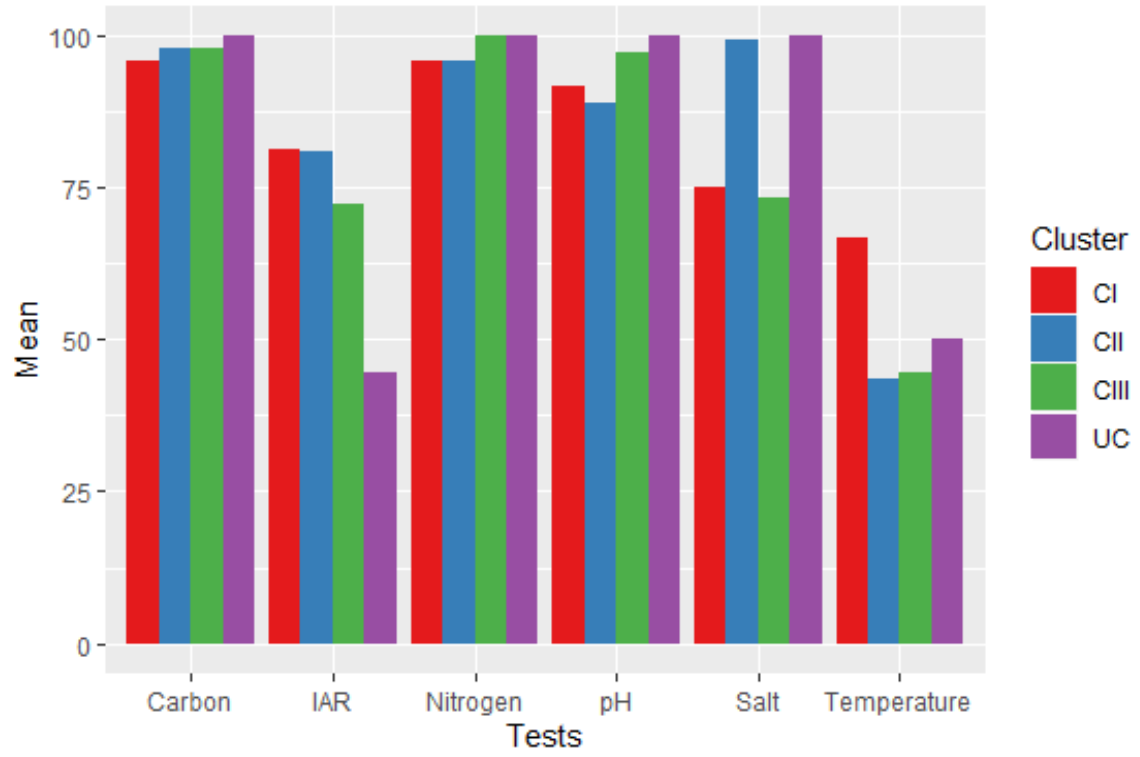

Figure 1performances of isolates in clusters to different tests

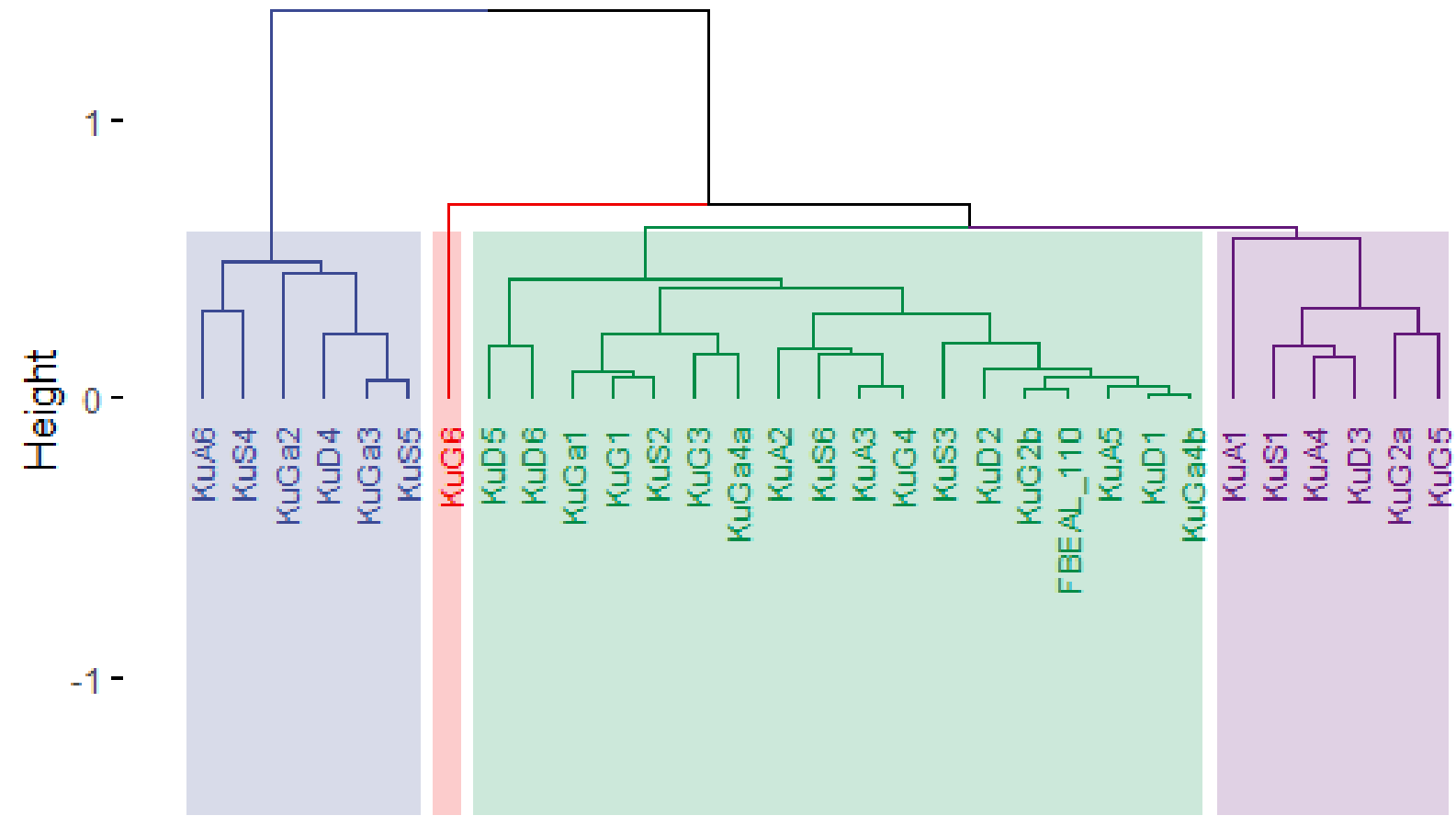

\section{rhizobial isolates}

Figure 2 dendrogram (based on Pearson correlation clustering method ) of isolates based on phenotypic similarities for Faba Bean Rhizobia (R.leguminosarum bv. viciae) from potential districts of Bale zone 


\section{Numerical Analysis}

Numerical classification by multivariate cluster analysis based on 56 characteristics(different levels of Temperature,Salt,pH,IAR,Carbon \&Nitrogen sources) produced isolates of different groups (Figure 2). The three clusters (CI,CII, and CIII) and one un clustered isolates (KUG6) were displayed. Six isolates fall into CI and CIII each and majority of isolates (58\%) including the reference strain were grouped in CIII.

CI (KUA6, KUS4, KUGa2, KUD4, KUGa3 and KUS5) had some important physiological characters and consistently performing at all tests similar to CII. Cluster CII (KUD5, KUD6, KUGa1,KUG1,KUS2,KUG3,KUGa4,KUA2,KUS6,KUA3,KUG4,KUS3,KUD2,KUG2b,FBEAL-

$110, \mathrm{KUA5}, \mathrm{KUD} 1$, and KUG4b) in which most of isolates grouped had some physiological characters that are unique to the cluster. Isolates in this cluster avoid extreme temperature and more than $95 \%$ of them limited themselves between temperature of $15^{\circ} \mathrm{C}$ to $35^{\circ} \mathrm{C}$ and remarkably grew at all salt $(0.1 \%$ to $6 \%)$ concentration. shares similarity to other clusters in utilization of sugar and nitrogen source $(100 \%)$.

CIII (KUA1, KUS4, KUA4, KUD3,KUG2a,KUG5) had some physiological characters that are not unique to the cluster. Isolates in this cluster avoid extreme temperature (like CII) and more than $95 \%$ of them limited themselves between temperature of $15^{\circ} \mathrm{C}$ to $35^{\circ} \mathrm{C}$ and grew at salt concentration similar to $\mathrm{CI}$. Concerning $\mathrm{pH}$, more tolerant to $\mathrm{pH}$ ranges that makes this cluster different from the other groups but like other clusters utilization to sugar and nitrogen sources is similar across all clusters. The other remaining one isolate(KUG6) was not clustered and had physiological characters that is quite extra ordinary in that to all tests tolerated different $\mathrm{pH}$,salt concentration and managed to utilize all Nitrogen and Carbon sources but IAR(streptomycin and chloramphenicol intolerant) and Temperature tolerance was susceptible and intolerant similar to other clusters (CI to CIII).

Rhizobia are influenced in edaphic situations of their micro environment. In such situations an increase in the antibiotic-resistant Rhizobia population and association with an increase in soil $\mathrm{P}$ and Al contents was revealed in studies of Xavier et al. (1998) on Faba Bean host and he further elaborated that isolates which were sensitive to spectinomycin, ampicillin, streptomycin, chloramphenicol and tetracycline were present at higher rates in soils devoid of Al. Relatively trends in growth observed to be affected when concentration increased and tetracycline was the least according to isolates performance at all concentration. Result is similar to the work of Zerihun Belay and Fassil Assefa (2011) and Girmaye Kenasa et al. (2014). Intrinsic antibiotic resistance and persistence as major factors to competition during natural invasion and it was stated that strains to vary in their success to cope up environmental contests and grow well on diverse antibiotics and loads (Tolera Abera et al., 2015).The relationship between total $P$ of the soil from which isolates trapped and that of IAR is not meaning full as stated but the presence of heavy metals like Al was not included in this study and need to be considered further with other heavy metals. In view of other eco-physiological characteristics(Figure 1) IAR was superior in indicating variability that might have put isolates into clusters.

Growth of isolates in a range of $\mathrm{pH}=4$ to 9 indicated that isolates grew in divers ranges . When this diversity is taken to clusters $\mathrm{CI}$ and CII are alike were found to be relatively unaffected to all $\mathrm{pH}$ ranges and $97 \%$ of CIII shares this feature but the un clustered isolate was total tolerant to all $\mathrm{pH}$ rangese. This trend in growth is similar to the work of Alemayehu Workalemahu (2009) and Getahun Negash (2015) in contrast to the assertion of Zerihun Belay and Fassil Assefa (2011) that general sensitivity to low $\mathrm{pH}$. The attribute indicated in morphology of colonies i.e. the mucus production observed in almost all of the isolates might have enabled to tolerate low pH.This manifestation can be taken as an adaptation of acid soils was reported in Brazilian tropical Savannah by Teixeira; et al. (2010). Girmaye Kenasa et al. (2014) emphasized strains resistant to different soil stresses such as $\mathrm{pH}$ have potential to improvement in the production of legumes grown on the area and extend the ranges of soils upon which legumes adapted to grow. And this diverse opportunity can be further utilized in future production of biofertilizers from which extreme condition tolerant strains may be tried and used.

Regarding stress test by different salt concentration $(0.1$ to $6 \%$ ), All isolates tolerated salt concentration of less than or equal to $1 \%$. When regarded through the clusters formed it was observed that all the three clusters and the un clustered isolated acted similarly to all salt concentration from 0.1 to $1 \%$. CI and CIII were not tolerant to some levels of salinity. CII In this case had some similarities with un clustered isolates were observed. These observations might highlight the concomitant rhizobium-host efficiency which is hampered by high levels of salinity which decreases the $\mathrm{Ca}^{2+}$ content of rhizobium cells, and the outer membrane structure of the rhizobium cells was greatly distorted (Zahran, 1999). In that regard Giller (2001) mentioned that salt stress limits legume growth, especially when the crop relies on symbiotically fixed nitrogen. But the response of both Rhizobia and host plant may differ and so a detrimental effect on soil microbial populations as a result of direct toxicity as well as through osmotic stress will be resulted. Simon et al. (2014) also reported in that some different combination of factors besides to salt and osmotic stress can affect the initial stage of legume-Rhizobial interaction and nodule formation. When it came to above $1 \%$ of concentration, even though further increment was not investigated it was observed that growth at higher salt concentration was in decrement trend which is similarly reported by the work of Zerihun Belay and Fassil Assefa (2011) and Amha Gebremariam and Fassil 
Assefa (2018).From this fact that the development of new nodules, the activity of nodules and the formation of the nitrogenase enzyme are reduced by salinity (Ahmed and Elsheikh, 1998) so the observed diversity in tolerance for these isolates has paramount importance.

During symbiosis ,temperature affects root hair infection, bacteroid differentiation, nodule structure, and the functioning of the legume root nodule and now a days several strains that tolerate higher temperature regime are being described (Zahran, 1999). Strains that could tolerate adverse conditions like elevated temperature were mentioned by Zahran (2001) in that case strains with this inherent characteristics exploited as a hot spot for future biotechnology tools. The cold adaptation observed, which is apparent because the source soil was from Bale zone which most of its districts under this investigation were humid to cool. It is worthy of further investigation with respect to the improvement of nitrogen fixation under cool climates and for studies on the mechanisms of cold adaptation (Pascal et al., 1996). Climatic predictions are apparent in that future trends are inhabitable and such resources with possessions of extreme stress tolerant make ups can be compromised. The work of Benidire et al. (2018) also highlighted abiotic stresses in his study to Moroccan Faba Bean that was produced in marginal lands .

Utilization in carbon and nitrogen source was assessed and isolates' pattern to utilization of sugar is very similar. Except very few(five) isolates which did not grow on either sorbitol or arabinose all isolates grew on almost all sugars. This trend also prevailed when the three clusters were explored and no noticeable variability was observed. This competence in nutrient utilization is useful trait in which the efficiency of the symbiosis is affected (Somasegaran and Hoben, 1994) by the levels of the various nutrients in the soil and variability in the response to inoculation. This similarity is corroborated with the work of Getahun Negash (2015) and Dereje Tsegaye et al. (2015) also in agreement with Zerihun Belay and Fassil Assefa (2011) who reported the isolates from North Gondar were found to utilize 80 to $100 \%$ of the tested monosaccharide and disaccharides, and similar claim with other researcher (Fano Berhe, 2010) on similar cross inoculating group. The results of utilization of nitrogen sources indicated that more than $96 \%$ of all isolates metabolized all the nitrogen sources which exhibited relative similarity with Girmaye Kenasa et al. (2014) in their work that all sources of nitrogen utilized which is in contrast to the work of Getahun Negash (2015) who stated that $37.1 \%$ of the isolates and commercial strains were able to metabolize all the nitrogen sources.

This nutritional and tolerance versatility is important in exploitation of any environment $\left(\mathrm{O}^{\prime} \mathrm{Hara}\right.$ et al., 2002). From this study, it was observed that strains isolated from the study area were diverse, and thus still there are untapped rhizobial resources in Ethiopian soils

\section{Refferences}

Abere Mnalku, Heluf Gebrekidan and Fassil Assefa (2009). Simbiotic effectivness and characterization of rhizobium strains of faba bean (Vicia faba L.) collected from Eastern and Wstern Hararghe highlands of Ethiopia. Ethiopian Journal of Natural Resources, 11(2):223-244.

Ahmed, A. E. and Elsheikh, E. A. E. (1998). Effects of Biological and Chemical Fertilizers on Growth and Symbiotic Properties of Faba Bean (Vida/aha L.) Under Salt Stress. University of Khartoum Journal of Agricultural science, 6(1):150-164.

Alemayehu Workalemahu (2009). The Effect of Indigenous Root-Nodulating Bacteria on Nodulation and Growth of Faba Bean (Vicia faba 1.) in the Low-Input Agricultural Systems of Tigray Highlands, Northern Ethiopia. Momona Ethopian Journal Of Science, 1(2):30-43.

Amha Gebremariam and Fassil Assefa (2018). The effect of inter cross-inoculation host group rhizobia on the growth and nitrogen fixation of Faba Bean (Vicia faba L.) varieties in North Showa,Amhara Regional State, Ethiopia. Journal of Agricultural Biotechnology and Sustainable Development, 10(2):25-33.

Benidire, L., Lahrouni, M., Daoui, K., Fatemi, Z. e. A., Gomez Carmona, R., Göttfert, M. and Oufdou, K. (2018). Phenotypic and genetic diversity of Moroccan rhizobia isolated from Vicia faba and study of genes that are likely to be involved in their osmotolerance. Systematic and Applied Microbiology, 41(1):51-61.

Berkum;, P. V., Beyene;, D., Vera;, F. T. and H.Keyser;, H. (1995). Variability among Rhizobium Strains Originating from Nodules of Vicia faba. African and Envronmental Microbiology, 61(7):2649-2653.

Dereje Tsegaye, Fasil Assefa, Heluf Gebrekidan and Gemechu Keneni, W. (2015). Nutritional, ecophysiological and symbiotic characteristics of rhizobia nodulating faba bean (Vicia faba L.) collected from acidic soils of Ethiopia. African Journal of Environmental Science and Technology, 9(7):646-654.

ECX (2009). Production \& Marketing Activity Of Broad Bean in Ethiopia.

Fano Berhe (2010). Phenotypic and Symbiotic characteristics of Rhizobia nodulating field Pea (Pisum sativum L.) in southern Tigray, Ethiopia. MSc. Thesis, Addis Ababa University.81 p.

Fisher, K. and Newton, W. E. (2002). Nitrogen Fixation- A General Overview. In: G. Jeffery Leigh (ed.) Nitrogen Fixation at the Millenniu. Elsevier.

Getahun Negash , T. (2015). Symbiotic and phenotypic characteristics of indigenous rhizobia nodulating faba bean (Vicia faba L.) growing in some parts of Wello, Northern Ethiopia. MSc thesis Hawassa 
University.111 p.

Giller, K. E. (2001). Nitrogen fixation in tropical cropping systems, CABI

Girmaye Kenasa, Jida, M. and Assefa, F. (2014). Characterization of Phosphate Solubilizing Faba Bean (Vicia faba 1.) Nodulating Rhizobia Isolated from Acidic Soils of Wollega, Ethiopia. Science,Technology and Arts Research, 3(3):11-17.

Howieson, J. G. and Dilworth, M. J. (2016). Working with rhizobia, Canberra,Australia, Australian Centre for International Agricultural Research.314.p.

IFPRI (2010). Pulses Value Chain in Ethiopia Constraints and Opportunities For Enhancing Exports, p.44.

Kassambara, A. (2017). Cluster Analysis in R, STHDA.187.p.

O'Hara, G. W., J.G.Howmson and P.H.Graham (2002). Nitrogen Fixation and Agricultural Practice. In: G. Jeffery Leigh (ed.) Nitrogen Fixation at the Millennium. Elsevier.

Pascal, D., Prevost, D. and Antoun, H. (1996). Classification of Bacteria Nodulating Lathyrus japonicus and Strains of Lathyrus pratensis in Northern Quebec as Rhizobium leguminosarum biovar viciae International Jornal of Systematic Bacteriology, 46(4):1016-1024.

R Core Team (2019). R: A language and environment for statistical computing. R Foundation for Statistical Computing, Vienna, Austria. URL https://www.R-project.org/. 3.6.1 ed.

Simon, Z., Mtei, K., Amare Gessesse and Ndakidemi, P. A. (2014). Isolation and Characterization of Nitrogen Fixing Rhizobia from Cultivated and Uncultivated Soils of Northern Tanzania. American Journal of Plant Sciences, 5:4050-4067.

Somasegaran, P. and Hoben, H. J. (1994). Handbook for Rhizobia:Methods in Legume-Rhizobium Technology, USA, Springer-Verlag.455.p.

Tamene Temesgen, Gemechu Keneni, Tadese Seferaa and Mussa Jarso (2015). Yield stability and relationships among stability parameters in faba bean (Vicia faba L.) genotypes. T he Crop Journal, 3:258 268.

Teixeira;, F. C. P. e., Borges;, W. L., Xavier;, G. R. and Rumjanek, N. G. (2010). Characterization of indigenous rhizobia from caatinga. Brazilian Journal of Microbiology, 41:201-208.

Tolera Abera, Semu, E., Debele, T., Wegar, D. and Kim, H. (2015). Determination Soil Rhizobium Populations, Intrinsic Antibiotic Resistance,Nodulation and Seed Yield of Faba Bean and Soybean in Western Ethiopia. World Journal of Agricultural Sciences, 11(5):311-324.

Xavier, G. R., L.M.V, M., M.C.P., N. and G., R. N. (1998). Edaphic factors as determinants for the distrbution of intrisic antibiotic resistance in a cow pea rhizobia population. Biology and Fertility of Soils, 27:386-392.

Zahran, H. H. (1999). Rhizobium-Legume Symbiosis and Nitrogen Fixation under Severe Conditions and in an Arid Climate. Microbiology And Molecular Biology Reviews, 63(4):968-989.

Zahran, H. H. (2001). Rhizobia from wild legumes: diversity, taxonomy, ecology,nitrogen fixation and biotechnology. Journal of Biotechnology, 91:143-153.

Zerihun Belay and Fassil Assefa (2011). Symbiotic and phenotypic diversity of Rhizobium leguminosarum bv. viciae from Northern Gondar,Ethiopia. African Journal of Biotechnology, 10(21):4372-4379. 